\title{
Mécanisme de la circulation perturbée du calcium et son traitement dans une cardiomyopathie dilatée (CMD) associée ou non ò une déficience en dystrophine (DMD)
}

\section{Dominique Mornet}

Résumé

Ce travail présente en détail le cas général d'une cardiomyopathie dilatée (CMD) avec un récapitulatif complet du cycle du calcium au niveau d'un muscle cardiaque dans un état normal [1], avec un catalogue des principales altérations protéiques possibles. Ces dernières résident dans le sarcomère et l'on y trouve l'actine, la tropomyosine, les troponines-C, I et T, la chaîne lourde de la myosine de type bêta et la titine, et, au niveau cytosquelettique, la desmine, la vinculine, la protéine ZASP et les sarcoglycanes. Cependant, une altération acquise soit par une infection de type viral, soit par une inflammation de type myocardite, peut également conduire à une cardiomyopathie. Dans le cadre particulier de la déficience en dystrophine (myopathie de Duchenne ou DMD), il est souvent observé une cardiomyopathie associée, et le présent article compare ces deux pathologies [1]. Indépendamment de l'origine, il est alors observé une dérégulation des flux calciques et les conséquences en sont analysées en détail. Cette revue fait le bilan exhaustif sur le double rôle de la dystrophine : protéine requise pour la stabilisation de l'intégrité membranaire, mais aussi impliquée dans l'organisation et le contrôle de la bonne distribution de divers partenaires en rapport avec le calcium et l'homéostasie oxydative de la cellule musculaire. On dispose ainsi d'un bilan sur les systèmes modèles permettant d'étudier comparativement les différences entre cardiomyopathie dilatée isolée et cardiomyopathie associée dans la myopathie de Duchenne, le dénominateur commun étant la dilatation myocardique, la fibrose et la mort de la cellule musculaire.

\section{Commentaire}

Cette analyse traite de ce qui est connu sur la base mécanique de la perturbation des flux calciques observés au cours de la CMD isolée, ceci en comparaison avec la cardiomyopathie observée chez le patient DMD. L'article passe en revue les stratégies thérapeutiques expérimentales ciblant les défauts des courants calciques observés au cours d'une CMD comme dans le cas de la cardiomyopathie associée à la $\mathrm{DMD}$. Un répertoire est présenté sur les thérapies déjà couramment initiées pour traiter ces deux types de pathologies cardiaques. On y trouve les corticostéroïdes et leurs effets cardioprotecteurs, les divers inhibiteurs concernant l'angiotensine, les molécules dites bêta-bloquantes du cœur, ou les antagonistes des récepteurs des minéralocorticoïdes. L'accent est mis sur le flux calcique avec le phospholamban (PLN), un régulateur du cycle calcique au niveau du cour. Dans le même ordre d'idée, une stimulation de l'expression de la protéine SERCA2a [2] ou de la mucolipine [3] semblent des stratégies thérapeutiques prometteuses. Par ailleurs, la parvalbumine, un capteur bien connu du calcium, permet d'envisager des interventions mettant en jeu d'autres capteurs du calcium comme les protéines S100 [4]. Les recherches visent à mieux comprendre le mécanisme initiateur d'une cardiomyopathie chez le patient DMD, et cela spécifiquement pour ceux liés à la perturbation des flux du calcium, pour permettre une approche ciblée vers des cibles thérapeutiques novatrices pour traiter de tels patients.

Mechanism of disturbed calcium circulation and its treatment in the general case of dilated cardiomyopathy (CMD) and associated with dystrophin deficiency (DMD patient)

\section{LIENS D'INTÉRÊT}

L'auteur déclare n'avoir aucun lien d'intérêt concernant les données publiées dans cet article.
Dominique Mornet Ancien DR2 CNRS,

PhyMedExp, Université de Montpellier, Inserm, CNRS, Montpellier 34295 Cedex 5 , France

Contact

domimornet@gmail.com

\section{RÉFÉRENCES}

1. Law ML, Cohen H, Martin AA, Angulski ABB, Metzger JM. Dysregulation of calcium handling in Duchenne muscular dystrophyassociated dilated cardiomyopathy: mechanisms and experimental therapeutic strategies. J Clin Med 2020 (sous presse).

2. Wasala NB, Yue Y, Lostal W, Wasala LP, Niranjan N, Hajjar RJ, Babu GJ, Duan D. Single SERCA2a therapy ameliorated dilated cardiomyopathy for 18 months in a mouse model of Duchenne muscular dystrophy. Mol Ther $2020 ; 283$ : 845-54

3. Yu L, Zhang X, Yang Y, Li D, Tang K, Zhao Z, He W, Wang C, Sahoo N, Converso-Baran K, Davis CS, Brooks S, Bigot A, Calvo R, Martinez NJ, Southall N, Hu X, Marugan J, Ferrer M, Xu H. Small-molecule activation of lysosomal TRP channels ameliorates Duchenne muscular dystrophy in mouse models. Sci Adv 2020 (sous presse).

4. Heizmann CW. $\mathrm{Ca}^{2+}$-binding proteins of the EF-hand superfamily: diagnostic and prognostic biomarkers and novel therapeutic targets. Methods Mol Biol 2019; 1929: 157-86. 